\title{
New applications of phonocardiography for the home-monitoring of cardiac patients
}

\author{
A Brensing, Fachbereich Ingenieurwissenschaften, Hochschule RheinMain, Ruesselsheim, Germany, \\ andreas.brensing@hs-rm.de
}

\section{Introduction}

The phonocardiogram implies the acoustic correlative of the mechanical incident inside the heart. Although displaced widely by imaging technology in clinical practice phonocardiography offers excellent prospects in the home-monitoring of cardiac patients. Here its application for the monitoring of heart valve prosthesis, chronic heart failure, and pulmonary hypertension is presented.

\section{Methods}

The acoustic phenomena of implanted mechanical heart valve prostheses were analysed in the time and frequency domain between $8 \mathrm{kHz}$ and $22 \mathrm{kHz}$ to evaluate the valve integrity. In the following 483 heart valve patients were included into a clinical trial on early detection of valve dysfunction. Secondly the ejection time was estimated from conventional phonocardiogram at chronic heart failure patients before and after intervention and correlated to cardiac performance. Thirdly the splitting and spectral pattern of second heart tone was investigated with respect to pulmonary hypertension. For all examinations a hand-held signal processing device was applied for sound recording and assessment.

\section{Results}

At heart valve patients it was possible to detect a thrombus formation by the loss of the characteristic sound pattern. In the clinical trial the sensitivity of this method was comparable to fluoroscopy and superior to echocardiography. The ejection time calculated from the duration between first and second heart tone corresponds to the values derived from carotid pulse wave analysis. At chronic heart failure patients the ejection time normalized to heart rate correlates to the ejection fraction in the intra-individual course. With respect to pulmonary hypertension the splitting and frequency spectrum of second heart tone is sensitive to the degree of morbidity.

\section{Conclusion}

Phonocardiography in terms of patient self-testing and telemetric data transfer enables the daily monitoring of cardiac performance in heart failure and pulmonary hypertension. At mechanical heart valve prostheses it can be used as an early warning system in case of thrombus formation. 